\title{
Over-Expression of Meteorin Drives Gliogenesis Following Striatal Injury
}

\author{
Jordan L. Wright ${ }^{1 *}$, Charlotte M. Ermine ${ }^{1}$, Jesper R. Jørgensen ${ }^{2}$, Clare L. Parish ${ }^{1}$ and \\ Lachlan H. Thompson ${ }^{1}$
}

${ }^{1}$ The Florey Institute of Neuroscience and Mental Health, The University of Melbourne, Melbourne, VIC, Australia, ${ }^{2}$ NsGene, Ballerup, Denmark

A number of studies have shown that damage to brain structures adjacent to neurogenic regions can result in migration of new neurons from neurogenic zones into the damaged tissue. The number of differentiated neurons that survive is low, however, and this has led to the idea that the introduction of extrinsic signaling factors, particularly neurotrophic proteins, may augment the neurogenic response to a level that would be therapeutically relevant. Here we report on the impact of the relatively newly described neurotrophic factor, Meteorin, when over-expressed in the striatum following excitotoxic injury. Birthdating studies using bromo-deoxy-uridine (BrdU) showed that Meteorin did not enhance injury-induced striatal neurogenesis but significantly increased the proportion of new cells with astroglial and oligodendroglial features. As a basis for comparison we found under the same conditions, glial derived neurotrophic factor significantly enhanced neurogenesis but did not effect gliogenesis. The results highlight the specificity of action of different neurotrophic factors in modulating the proliferative response to injury. Meteorin may be an interesting candidate in pathological settings involving damage to

OPEN ACCESS

Edited by:

Alessandro Tozzi,

University of Perugia, Italy

Reviewed by:

Afsaneh Gaillard,

University of Poitiers, France

Valentina Pendolino,

University College London, UK

${ }^{*}$ Correspondence:

Jordan L. Wright

jordan.wright@ucl.ac.uk

Received: 19 February 2016 Accepted: 23 June 2016

Published: 05 July 2016

Citation:

Wright JL, Ermine CM, Jørgensen JR, Parish CL and

Thompson LH (2016)

Over-Expression of Meteorin Drives

Gliogenesis Following Striatal Injury.

Front. Cell. Neurosci. 10:177.

doi: 10.3389/fncel.2016.00177 white matter, for example after stroke or neonatal brain injury.

Keywords: forebrain injury, striatum, neurogenesis, oligodendrogenesis, GDNF, brain repair

\section{INTRODUCTION}

Meteorin is a member of a newly described family of secreted proteins that also includes the related protein, Meteorin-like. It is highly expressed in the mammalian brain, throughout midto late-development and is maintained at lower levels in the post-natal brain (Jorgensen et al., 2009). In adult rodents, Meteorin protein is most conspicuously expressed by Bergmann glia in the cerebellum, but also more diffusely at lower levels in glia cells throughout the brain. It was first characterized in vitro for its ability to promote astrocyte differentiation and axon growth in neurosphere cultures and dorsal root ganglion explants, respectively, (Nishino et al., 2004). Later studies established that Meteorin's pro-gliogenic functionality acted on the Jak-STAT3 pathway (Lee et al., 2010; Sun et al., 2014), although to date, the receptor it functions through remains unknown.

In vivo studies in rodents demonstrated that after excitotoxic injury to the striatum, endogenous Meteorin is up-regulated in striatal s100 $\beta^{+}$astrocytes (Jorgensen et al., 2011), and has also been observed in neurons after ischaemic damage (Wang et al., 2012). To explore it's functional impact in this setting, subsequent studies demonstrated that over-expression of Meteorin via viral (Jorgensen et al., 2011) or encapsulated cell (Tornoe et al., 2012) delivery prior to excitotoxic injury resulted in significantly reduced levels of striatal damage (Jorgensen et al., 2011; Tornoe et al., 2012). More recently, studies have also begun to investigate the possibility that, in addition to neuroprotection 
of existing circuitry, Meteorin might also act as a survival factor for newborn neurons generated in response to striatal injury (Wang et al., 2012).

A number of studies have shown that, following striatal injury resulting in extensive cell loss, neuroblasts constitutively generated in the sub-ventricular zone (SVZ) can migrate into the damaged striatal parenchyma and differentiate into mature neurons (Arvidsson et al., 2001, 2002; Jin et al., 2001, 2003, 2006; Parent et al., 2002). The overall survival of these new neurons is notably poor and well below levels likely to be required for therapeutic replacement of neurons lost to the injury. This has motivated studies that investigate strategies to improve the survival of newborn neurons, for example through delivery of trophic factors. Topical delivery of soluble proteins known to influence cell survival, neural stem cell proliferation and neurogenesis in other disease models have proven to be robust regulators of these cellular processes in the context of forebrain injury. Examples of such factors are Glia-derived neurotrophic factor (GDNF; Kobayashi et al., 2006), Vascular endothelial growth factor (VEGF; Sun et al., 2003), along with Noggin and Brainderived neurotrophic factor (BDNF; Benraiss et al., 2012) which have all been shown to positively regulate neurogenesis in the damaged striatum. The expression of Meteorin during neural development and in the adult striatum makes it an interesting candidate in this context. Recently, Wang et al. (2012) demonstrated that chronic infusion of Meteorin increased SVZ proliferation and neuroblast migration into the damaged striatum after medial cerebral artery occlusion in rats, resulting in increased numbers of surviving newborn neurons.

Here we sought to characterize the phenotype of new cells generated in the presence of Meteorin after striatal damage - including the identity of neuronal and glial subtypes. Following excitotoxic damage, we found that stable expression of Meteorin using a lenti-virus resulted in a small increase in the survival of new neurons which did not reach statistical significance. To contextualize this impact on neuronal survival, we also present results from GDNF expression in the same experimental paradigm, where there was a much larger effect on neurogenesis in response to injury. The major impact of Meteorin was in fact to drive gliogenesis, where birth-dating studies using Bromodeoxyuridine (BrdU) showed that there was a significant increase in the proportion of new $s 100 \beta^{+}$ astrocytes and Oligodendrocyte transcription factor $2\left(\mathrm{Olig}^{+}\right)$ oligodendrocyte progenitors in response to damage to the adult striatum.

\section{MATERIALS AND METHODS}

\section{Ethical Approval}

All experimental procedures carried out in this study conform to the rules set out by the Australian National Health and Medical Research Council published code of practice and experiments were approved by the Florey Neuroscience Institutes animal ethics committee (no. 09-105).

\section{In Vivo Procedures}

Adult Sprague-Dawley rats were housed under a 12-h light/dark cycle with ad libitum access to food and water. Surgery was performed at 6 weeks of age using age matched animals. Prior to surgery, rats were deeply anesthetized with isoflurane $(5 \%$ at $1 \mathrm{~L} / \mathrm{min})$ and kept under anesthesia $(2-3 \%$ at $1 \mathrm{~L} / \mathrm{min})$ for the duration of surgery.

All surgical procedures were performed using a stereotaxic frame (Kopf, Germany) and intra-striatal delivery was performed using a fine glass capillary fitted to a $5 \mu \mathrm{l}$ microsyringe (SGE Analytical Sciences, Australia). Under deep anesthesia, all animals received $2 \mu \mathrm{l}$ of quinolinic acid (QA) solution ( $100 \mathrm{nmol} / \mu \mathrm{l}$ in $0.9 \%$ saline) injected over $2 \mathrm{~min}$ into the striatum (1.2 $\mathrm{mm}$ anterior and $3 \mathrm{~mm}$ lateral to bregma and $4 \mathrm{~mm}$ below the dural surface). The cannula was kept in place for 5 min after injection to minimize back-flow.

Twenty four hours post-injection, all animals underwent a second round of surgery (setup as previously described) for intrastriatal lentivirus delivery constructed as previously described (Jorgensen et al., 2011). Three treatment groups were compared within this study. Animals received one of either lentivirus encoding Meteorin (Jorgensen et al., 2011), GDNF (pHsCXW, NsGene), and GFP (pHsCXW, NsGene) for control animals via a $1.5 \mu$ l solution $\left(1.2 \times 10^{5}\right.$ transforming units total $)$ over $2 \mathrm{~min}$ into the striatum $(1.2 \mathrm{~mm}$ anterior and $3.2 \mathrm{~mm}$ lateral to bregma and $4 \mathrm{~mm}$ below the dural surface). All vectors were under the control of the woodchuck post-regulatory element for constitutive transgene expression.

To label dividing cells, animals received BrdU (50 mg/kg i.p. using a $20 \mathrm{mg} / \mathrm{ml}$ solution in $0.9 \%$ saline) every $12 \mathrm{~h}$ for 2 weeks as previously described (Arvidsson et al., 2002), beginning the day of lentiviral delivery. Animals were perfused for histological assessment 4 weeks after BrdU treatment (6 weeks after QA lesion).

\section{Tissue Preparation}

At 6 weeks after surgery, animals under deep anesthesia induced with isoflurane $(5 \%$ at $1 \mathrm{~L} / \mathrm{min})$, subsequently received a lethal dose of pentobarbitone. Animals were then transcardially perfused with $50 \mathrm{ml}$ saline $(0.9 \% \mathrm{w} / \mathrm{v})$ followed by $200-250 \mathrm{ml}$ paraformaldehyde (PFA; $4 \% \mathrm{w} / \mathrm{v}$ in $0.1 \mathrm{M} \mathrm{PBS})$. The brains were removed, post-fixed a further $2 \mathrm{~h}$ in PFA and cryoprotected in sucrose $(20 \% \mathrm{w} / \mathrm{v}$ in $0.1 \mathrm{M}$ PBS). Brains were sectioned in the coronal plane in a 1:12 series at a thickness of $30 \mu \mathrm{m}$ on a freezing microtome (Leica, Germany).

\section{Immunohistochemistry}

Primary antibodies were diluted in a solution of $0.1 \mathrm{M}$ PBS containing 5\% normal serum and $0.5 \%$ Triton X-100 (Amereso, USA) and incubated with sections overnight at room temperature. Prior to the addition of the secondary antibody solution, the sections were blocked with $5 \%$ normal serum for 0.5-1 h. Secondary antibodies were diluted in a solution of $0.1 \mathrm{M}$ PBS containing 2\% normal serum and $0.5 \%$ Triton X-100 and incubated for $2 \mathrm{~h}$ at room temperature. For fluorescent analysis, secondary antibodies were conjugated to Dylight Fluorophores 
488 , 549, or 647 and slide-mounted sections were coverslipped with fluorescent mounting medium (DAKO, USA). For chromogenic analysis, secondary antibodies were conjugated to biotin and visualized using the Avidin/Biotin enzyme complex (VECTASTAIN ABC system, Vector Labs) coupled with peroxidase-driven precipitation of diaminobenzidine (DAB). DAB-labeled sections were dehydrated in alcohol and xylene, and cover-slipped with DePex mounting medium (BDH Chemicals, UK). For BrdU labeling, free-floating sections were incubated for $2 \mathrm{~h}$ at $65^{\circ} \mathrm{C}$ in $50 \%$ de-ionized formamide in $0.1 \mathrm{M}$ PBS. The tissue was rinsed $3 \times 5 \mathrm{~min}$ washes in $0.1 \mathrm{M}$ PBS and acid treated with $2 \mathrm{M} \mathrm{HCL}$ for $30 \mathrm{~min}$ at $37^{\circ} \mathrm{C}$. The tissue was then washed in 0.1 M Sodium Borate ( $\mathrm{pH} 8.5$ ) for $15 \mathrm{~min}$ and finally rinsed with $3 \times 5$ min washes in $0.1 \mathrm{M}$ PBS.

Primary antibodies and dilution factors were as follows: mouse anti-APC (1:200; \#OPT80, Calbiochem, Germany), rat anti-BrdU (1:300; \#OBT0030, Axyll Laboratories, USA), mouse anti-Calretinin (1:1000; \#7697, Swant, Switzerland), rabbit antiDarpp32 (1:500; \#AB10518, Millipore, Germany), goat antiGDNF (1:200; \#AB212NA, R\&D, USA), chicken anti-GFP (1:1000; AB13970, AbCam, USA), rabbit anti-GFP (1:20,000; \#AB13970, AbCam, USA), rabbit anti-Iba1 (1:200; \#01919741, Wako, Japan), goat anti-Meteorin (1:400; \#AF3475, R\&D, USA), mouse anti-NeuN (1:200; \#MAB377, Millipore, USA), rabbit anti-Olig2 (1:200; \#AB9610, Millipore, USA), rabbit anti-s100ß (1:200; \#HPA015768, Sigma-Aldrich, USA).

\section{Imaging}

Images of representative co-labeled cells were acquired via $40 \times$ or $63 \times$ PL-APO oil immersion objectives (Zeiss) using a Zeiss 780 laser-scanning confocal upright microscope. Representative overview images of DAB stained sections were acquired on a Leica DM6000B microscope using a HCX PL $5 \times / 0.5$ objective and tiled images aligned using Leica application suite v3.8 software. NeuN, BrdU, Iba1, Meteorin, GDNF and GFP immuno-labeled DAB representative images were acquired on the same microscope using HCX PL $20 \times / 0.5$ and HCX FL PLAN $40 \times / 0.65$ objectives (Leica). The brightness and contrast of individual images was adjusted using the photo enhancing software Photoshop v7.0 (Adobe) to optimally represent the immunohistochemistry observed through the microscope.

\section{Stereology and BrdU+ Cell Density Estimation}

Stereological estimation was used to determine the number of $\mathrm{BrdU}^{+}$cells residing in the anterior head of the striatum across the three treatment groups. For all animals, $30 \mu \mathrm{m}$ coronal sections each $360 \mu \mathrm{m}$ apart (1:12 series) were immunolabeled for BrdU. To establish the region of interest, the rostral boundary of striatum was defined at $1.70 \mathrm{~mm}$ anterior to bregma and the caudal boundary defined at $0.26 \mathrm{~mm}$ anterior to bregma (spanning $0-1440 \mu \mathrm{m}$ of the anterior striatum) and delineated by standard anatomical boundaries (Paxinos and Watson, 1998). Estimates of BrdU-labeled cells were determined using a fractionator sampling design according to optical dissector rules (Gundersen et al., 1988; Mayhew, 1991). Counting frame grid dimensions and fractionator $\mathrm{x}, \mathrm{y}$ coordinates were determined using the grid overlay program Stereoinvestigator v7.0 (MicroBrightField, Williston, VT, USA, used on a microscope, Leica, with a $40 \times$ objective, Zeiss). Guard zones were set at $1 \mu \mathrm{m}$ (top and bottom) and BrdU-labeled nuclei quantified within the counting frame (dimensions used were $90 \mu \mathrm{m} \times 90 \mu \mathrm{m}$ ) at periodic intervals $(\mathrm{x}=300 \mu \mathrm{m}, \mathrm{y}=350 \mu \mathrm{m})$ in the delineated region of interest.

To determine BrdU density, volumetric analysis was conducted to determine the volume of measured striatum. Striatal volumetric measurements for each brain were achieved via Cavalieri estimation using the program Stereoinvestigator v7.0 (MicroBrightField, Williston, VT, USA). The resultant volume was used to determine the $\mathrm{BrdU}^{+}$cell density in the striatum based on the stereological estimation of $\mathrm{BrdU}^{+}$cells and reported as $\mathrm{BrdU}^{+} / \mathrm{mm}^{3}$. The accuracy of the stereological estimations was determined by the coefficients of error and coefficients of variance. Estimations were deemed acceptable if coefficients were $>0.1$ (West et al., 1991).

\section{Cell Quantification}

In order to assess the phenotype of newborn cells post-lesion in the striatum, sections from animals sacrificed at 6 weeks post-lesion were double or triple labeled using fluorescent immunohistochemical techniques. Sections were stained with either BrdU/NeuN/Darpp32, BrdU/NeuN/Calretinin, $\mathrm{BrdU} / \mathrm{s} 100 \beta$ or BrdU/Olig2/APC. Due to the relatively low number of double and triple labeled cells in the striatum, cells were manually counted across 3 sections spanning the anterior head of the striatum $(1.70,1.34$, and $0.98 \mathrm{~mm}$ anterior to bregma). An additional rostral striatal section was analyzed at $0.62 \mathrm{~mm}$ from bregma for quantification of $\mathrm{BrdU}^{+} / \mathrm{s} 100 \beta^{+}$ co-localization.

Using a confocal microscope (Zeiss) and a $20 \times / 0.8 \mathrm{PL}-\mathrm{APO}$ objective (Zeiss), $\mathrm{z}$-stacking, and $\mathrm{x}-\mathrm{y}$ tile scanning features were used to capture large 3-dimensional volumes of the entire striatum for each tissue section. ZEN digital imaging software (Zeiss) was then used to unambiguously identify all double/triple labeled $\mathrm{BrdU}^{+}$cells. When delineating the striatal volume for analysis, we did not include the highly damaged area immediately in the vicinity of the QA delivery, which we found to have a high level of brightly fluorescent nonspecifically labeled cells. All $\mathrm{BrdU}^{+}$cells initially identified as co-labeled, were verified by inspection with a $63 \times$ PL-APO oil immersion objective (Zeiss) and orthogonal reconstruction on the $\mathrm{z}$-axis.

The total number of cells counted across the quantified striatum ( $1.70 \mathrm{~mm}-0.98 \mathrm{~mm}$ anterior to bregma) was estimated by extrapolation based on the series interval (1:12) and the number of sections counted (3) across the series. In addition to reporting total cell counts, total cell counts were normalized to $\mathrm{BrdU}^{+}$cell density to account for any variability in these parameters across animals. This normalized result is reported in the text as a percentage of $\mathrm{BrdU}^{+}$cell density $\left(\mathrm{BrdU}^{+} / \mathrm{mm}^{3}\right)$. 
For newborn $\mathrm{Iba1}^{+}$microglia quantification, sections were stained with BrdU/Iba1. Due to the abundance of $\mathrm{BrdU}^{+} / \mathrm{Iba1}^{+}$ cells, one section per animal across treatment groups $(n=3$ per group) was used for quantification ( $1.34 \mathrm{~mm}$ anterior to bregma). Striatal sections were systematically analyzed from the lateral ventricle to the site of injury across all animals for consistency. $\mathrm{BrdU}^{+}$cells and $\mathrm{BrdU}^{+} / \mathrm{Ibal}^{+}$cells were quantified in this manner. The resultant $\mathrm{BrdU}^{+} / \mathrm{Iba1}^{+}$cell counts were normalized to the $\mathrm{BrdU}^{+}$cell counts obtained from the same experiment and represented as a percentage.

\section{Statistics}

Statistical analysis was conducted using Graphpad Prism v6.0 software. BrdU ${ }^{+}$cell density, total cell counts and normalized cell counts were compared across the three experimental treatment groups using one-way ANOVA with post hoc correction (Bonferroni) for multiple comparison. In this study the data across experimental treatment groups is reported as the mean \pm SEM.

\section{RESULTS}

\section{Neuronal Cell Loss and Local Proliferation after Excitotoxic Lesioning}

Immunohistochemical labeling of the mature neuronal marker NeuN showed that injection of QA into the anterior head of the striatum resulted in a robust loss of neurons (Figures $\mathbf{1 A}, \mathbf{B}$ ). In some cases we also observed neuronal loss in the overlying cortex, likely resulting from reflux along the injection tract (not shown). Labeling for BrdU showed a robust proliferative response during the first 2 weeks after the lesion (Figures 1C,D). The $\mathrm{BrdU}^{+}$ cells were distributed densely in the immediate vicinity of the QA injection site, along with what appeared to be some degree of non-specific labeling, and also more diffusely throughout the entire striatum. The excitotoxic lesion also resulted in an inflammatory response that was persistent at 6 weeks, as demonstrated by a large increase in the density of striatal Iba1 ${ }^{+}$ microglia with a reactive, amoeboid morphology (Figures 1E,F). Some degree of microglial activation was also observed along



FIGURE 1 | Assessment of lesioning, proliferation, and microglial response 6 weeks after intra-striatal injection of quinolinic acid (QA). Representative striatal sections of intact and lesioned brains 6 weeks post-lesion (arrow indicates injection site of QA lesion). The boxed area on each section corresponds to the adjacent $20 \times$ image detailing the immuno-labeling. (A) NeuN labeling in intact striatum and (B) 6 weeks post-lesion, detailing loss of neurons in adjacent $20 \times$ image. (C) BrdU labeling of an intact adult striatal section and (D) 6 weeks post lesion. (E) Iba1 staining in intact sections with adjacent $20 \times$ image detailing ramified microglia in the striatum and at (F) 6 weeks post-lesion with reactive microglia illustrated in the adjacent $20 \times$ image. Scale bar: 1 mm brain sections (A-F), $200 \mu \mathrm{m}$ boxed images (A-F). 
the needle tract in the overlying cortex. Anatomically, the distribution of microglial activation matched well with the area of $\mathrm{NeuN}^{+}$cell loss and increased $\mathrm{BrdU}^{+}$labeling.

\section{Meteorin Is Highly Diffusible Within the Striatum When Over-Expressed Using a Lentiviral Vector}

Transduction of the striatum using lentiviral vectors carrying either GFP (lvGFP), GDNF (lvGDNF) or Meteorin (lvMeteorin) resulted in robust expression of the transgenes (Figures $\mathbf{2 A - F}$ ). At 6 weeks, immunohistochemical analysis showed the expected cytoplasmic pattern of GFP expression (Figure 2B). The morphology of the $\mathrm{GFP}^{+}$cells showed that predominantly astrocytes were transduced with the vector, although we observed a smaller population of $\mathrm{GFP}^{+}$neurons also. Injection of either lvMeteorin or lvGDNF resulted in a robust expression throughout the entire striatum (Figures 2C,E). In both cases, the profile of expression was consistent with that expected for a secreted protein, including a diffuse labeling pattern that reduced in intensity with distance from the injection site (Figures 2D,F).

\section{Over-Expression of Meteorin Did Not Increase the Number of New Neurons in the Striatum after Excitotoxic Damage}

To assess the impact of Meteorin on neurogenesis in response to injury, animals were administered BrdU twice daily for
2 weeks to label newborn cells immediately after excitotoxic damage to the striatum. Animals were perfused for histology 4 weeks later to allow for cell migration and differentiation of BrdU-labeled cells. Stereological estimation of the total number of newborn $\mathrm{BrdU}^{+}$cells in the striatum 6 weeks after injury showed no significant difference in the density of $\mathrm{BrdU}^{+}$cells in the striatum between the lvGFP control $\left(1.8 \pm 0.3 \times 10^{4} \mathrm{BrdU}^{+}\right.$ cells $\left./ \mathrm{mm}^{3}\right)$, lvMeteorin $\left(1.2 \pm 0.1 \times 10^{4} \mathrm{BrdU}{ }^{+}\right.$cells $\left./ \mathrm{mm}^{3}\right)$ and lvGDNF $\left(2.7 \pm 0.9 \times 10^{4} \mathrm{BrdU}^{+}\right.$cells $\left./ \mathrm{mm}^{3}\right)$ groups $(p=0.67$, lvGFP v lvGDNF; $p=0.95$, lvGFP v lvMeteorin, one-way ANOVA, Bonferroni post hoc - Figure 3A), although the number of $\mathrm{BrdU}^{+}$cells was notably variable in the lvGDNF group.

To estimate the number of new neurons generated during the 2 week BrdU-labeling period after striatal injury, cells unambiguously immuno-labeled for both BrdU and NeuN were counted in three coronal sections from each animal 4 weeks after the final BrdU injection (6 weeks after injury). This involved a first screening whereby every $\mathrm{BrdU}^{+}$cell was inspected for evidence of double labeling, followed by a second level of screening where double labeling was confirmed by confocal analysis, including orthogonal reconstruction on the $\mathrm{z}$-axis (Figures 3E,F).

Comparison of the total $\mathrm{BrdU}^{+} / \mathrm{NeuN}^{+}$cell counts revealed that the lvGFP control group (86.6 \pm 48.4 cells) was not significantly different in newborn mature neurons compared to lvMeteorin treated animals ( $88.5 \pm 32.6$ cells), but a significant increase was detected in the lvGDNF group (696 \pm 116.2



FIGURE 2 | Validation of lentiviral expression 6 weeks post-lesion. Immunohistochemical analysis at 6 weeks post-injury in animals administered corresponding lentiviral over-expression constructs across three representative sections (arrow indicates injection site of lentivirus) (A,C,E) corresponding to 1.70, 1.34 and $0.98 \mathrm{~mm}$ from bregma, left to right. (A) IvGFP treated animals with GFP immuno-labeling with (B) $40 \times$ representative image of GFP ${ }^{+}$astrocytic cells in the striatum. (C) IVMeteorin and (E) IVGDNF treated animals with Meteorin and GDNF immuno-labeling, respectively. (D) Tiled 40x image of IVMeteorin treated animals and (F) IvGDNF treated animals illustrate diffuse labeling within the striatum. Scale bar: $1 \mathrm{~mm}$ (A) analogous for (C,E), $0.1 \mathrm{~mm}$ (B,D,F). 
A

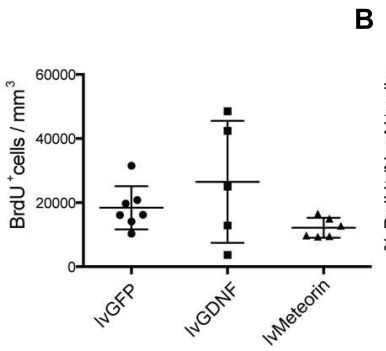

B
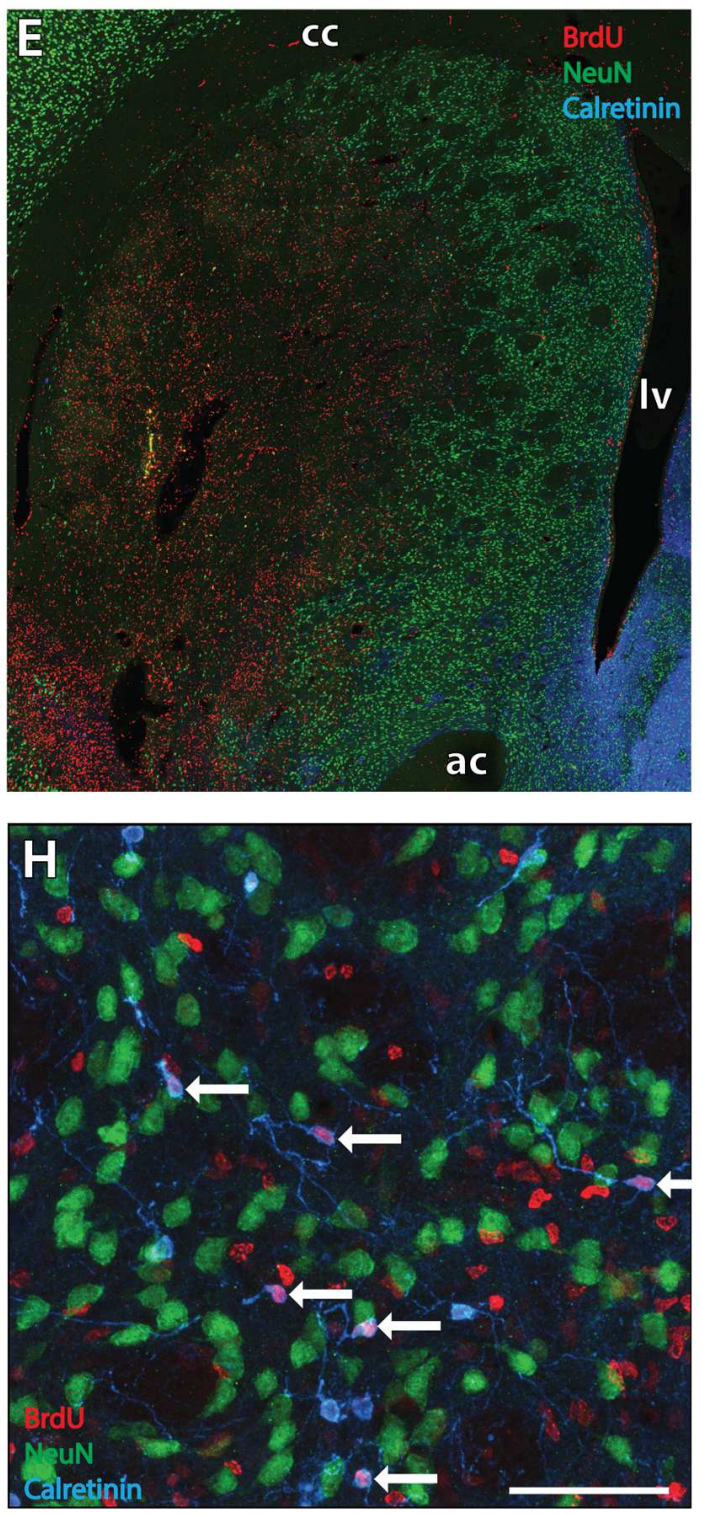

C
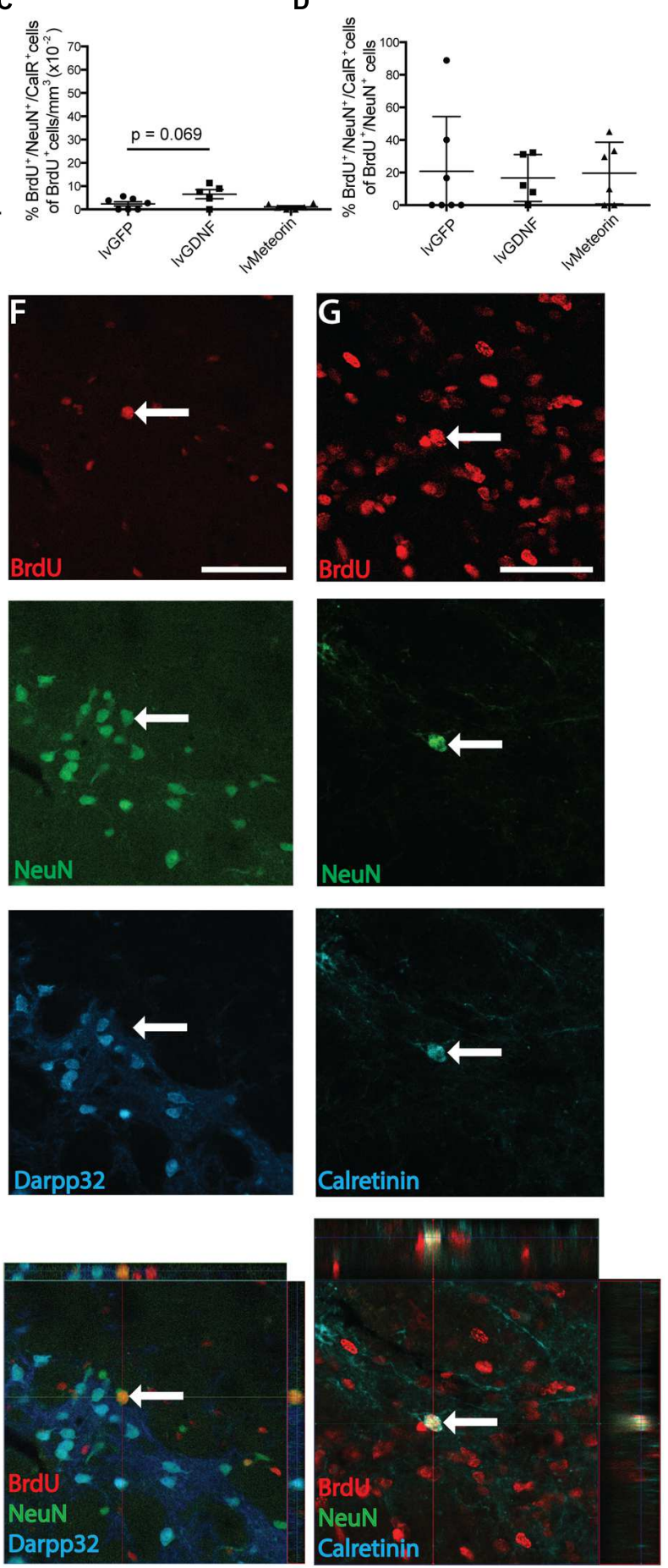

FIGURE 3 | Birth-dating of striatal neurons after quinolinic acid lesion to assess the effect of GDNF and Meteorin on these populations.

(A) Quantification of $\mathrm{BrdU}^{+}$cell density across treatment groups revealed no significant change in newborn cells in the striatum. (B) Quantification of BrdU+ ${ }^{+}$cells co-expressing NeuN across treatment groups normalized to BrdU ${ }^{+}$cell density revealed IvGDNF increased the number of new neurons in the ipsilateral striatum, with IvMeteorin having no effect compared to IvGFP controls. (C) Quantification of $\mathrm{BrdU}^{+} / \mathrm{NeuN}^{+} / \mathrm{CalR}^{+}$cells normalized to BrdU ${ }^{+}$cell density identified IvGDNF treatment trended toward an increase in this population. (D) CalR ${ }^{+}$cells normalized to total $\mathrm{BrdU}^{+} / \mathrm{NeuN}^{+}$cells indicated differentiation into this phenotype is altered across all newborn neurons. Overview image of BrdU/NeuN/CalR staining (E) and representative orthogonal z-stack confocal images of immuno-labeled (F) $\mathrm{BrdU}^{+} / \mathrm{NeuN}^{+} / \mathrm{Darpp} 32^{-}$neurons and (G) $\mathrm{BrdU}^{+} / \mathrm{NeuN}^{+} / \mathrm{CalR}^{+}$interneurons (arrow). (H) NeuN negative interneurons were also observed as a primary subset of $\mathrm{BrdU}^{+} /$Calretinin ${ }^{+}$cells (arrows). Data represents mean \pm SEM. IvGFP $n=7$; IvMeteorin $n=6$; IvGDNF $n=5$. Scale bars: $100 \mu \mathrm{m}$. cc, corpus callosum; Iv, lateral ventricle; ac, anterior commissure; CalR, Calretinin. 
cells, $p=<1 \times 10^{-4}$, one-way ANOVA, Bonferroni post hoc). To account for variability in BrdU dosage and striatal volume between animals and to assess the proportion of newborn cells within the striatum acquiring a mature neuronal phenotype, we normalized the total number of $\mathrm{BrdU}^{+} / \mathrm{NeuN}^{+}$ cells to $\mathrm{BrdU}^{+}$cell density $\left(\mathrm{BrdU}^{+}\right.$cells $\left./ \mathrm{mm}^{3}\right)$ to acquire a percentage (Figure 3B). In lvGFP-treated controls a low percentage of newborn cells post-lesion obtained a $\mathrm{NeuN}^{+}$ phenotype $\left(5.3 \pm 0.2 \times 10^{-2} \%\right.$ of $\mathrm{BrdU}^{+}$cells $\left./ \mathrm{mm}^{3}\right)$. This was not significantly different to lvMeteorin-treated animals $\left(8.5 \pm 0.4 \times 10^{-2} \%\right.$ of $\mathrm{BrdU}^{+}$cells $\left./ \mathrm{mm}^{3}\right)$ but in the lvGDNF-treated group, an 11-fold increase in the percentage of $\mathrm{BrdU}^{+} / \mathrm{NeuN}^{+}$cells was detected $\left(47.1 \pm 0.7 \times 10^{-2} \%\right.$ of $\mathrm{BrdU}^{+}$ cells $/ \mathrm{mm}^{3}, p=<1 \times 10^{-4}$, one-way ANOVA, Bonferroni post hoc; see Figures 3E,F for examples of immunohistochemistry).

Immunohistochemical co-labeling of $\mathrm{BrdU}^{+} / \mathrm{NeuN}^{+}$cells with markers for striatal projection neurons (Darpp32) or certain interneuron subtypes (Calretinin) allowed us to assess the impact of Meteorin or GDNF over-expression on the phenotype of newly generated neurons following injury. Although previous studies have indicated a proportion of new neurons generated after injury acquire a Darpp $32^{+}$identity at (Arvidsson et al., 2002; Parent et al., 2002), we did not observe any Darpp $32^{+} / \mathrm{BrdU}^{+}$cells across the three treatment groups. Some of the $\mathrm{BrdU}^{+} / \mathrm{NeuN}^{+}$cells were found to express Calretinin. Looking at $\mathrm{BrdU}^{+} / \mathrm{NeuN}^{+} /$Calretinin ${ }^{+}$cells as a percentage of total $\mathrm{BrdU}^{+}$cells $/ \mathrm{mm}^{3}$, there was no significant difference across the three treatment groups although there was a trend toward a greater proportion in the lvGDNF group $\left(6.5 \pm 0.2 \times 10^{-2}\right.$ $\%$ of $\mathrm{BrdU}^{+}$cells $/ \mathrm{mm}^{3}$ ) compared to the lvGFP control group $\left(2.4 \pm 0.1 \times 10^{-2} \%\right.$ of $\mathrm{BrdU}^{+}$cells $/ \mathrm{mm}^{3} ; p=0.069$, oneway ANOVA, Bonferroni post hoc) - Figures 3C,G. Similarly, as a percentage of total newborn neurons ( $\mathrm{BrdU}^{+} / \mathrm{NeuN}^{+}$cells) we observed similar proportions of cells obtaining Calretinin ${ }^{+}$ phenotype between the lvGFP ( $20.8 \pm 12.7 \%$ of cells $)$, lvMeteorin (19.6 $\pm 7.7 \%$ of cells) and lvGDNF groups $(16.7 \pm 6.4 \%$ of cells) - Figure 3D. We also observed across all groups numerous examples of BrdU+/Calretinin + cells with aspiny neurites that did not express NeuN within the damaged striatum (Figure $3 \mathbf{H}$ ). This may be indicative of a more immature population of newborn interneurons.

\section{Meteorin Increases Gliogenesis Following Striatal Damage}

Co-labeling of $\mathrm{BrdU}^{+}$cells with $\mathrm{s} 100 \beta$ or Olig2 showed that Meteorin over-expression significantly increased the proportion of $\mathrm{BrdU}^{+}$cells with astrocyte and oligodendroglial progenitor phenotypes, respectively.

Six weeks after injury, quantification of $\mathrm{BrdU}^{+} / \mathrm{s} 100 \beta^{+}$cells in the lvGFP control group showed that, as a fraction of $\mathrm{BrdU}^{+}$cell density, $1.3 \pm 0.3 \%$ were identified as $\mathrm{s} 100 \beta^{+}$ astrocytes (Figures $\mathbf{4 A}, \mathbf{C}$ ). One-way ANOVA showed that the average fraction of $\mathrm{BrdU}^{+}$cells co-labeled with $\mathrm{s} 100 \beta$ was not significantly different in the lvGDNF group $(0.9 \pm 0.3 \%)$ but was significantly greater in animals treated with lvMeteorin $(4.6 \pm 1.5$ $\% ; p=0.048$, one-way ANOVA, Bonferroni post hoc; Figure 4C).
To look at the effects of treatment on oligodendrocyte lineage cells, cells were co-labeled for $\mathrm{BrdU}^{+}$with the pan oligodendrocyte marker Olig2 and the post-mitotic oligodendrocyte marker APC. In all treatment groups we observed examples of $\mathrm{BrdU}^{+} / \mathrm{Olig}^{+}$cells that were both positive and negative for APC expression, indicative of oligodendrocyte progenitors $\left(\mathrm{BrdU}^{+} / \mathrm{Olig}^{+} / \mathrm{APC}^{-}\right)$and more mature oligodendrocytes $\left(\mathrm{BrdU}^{+} / \mathrm{Olig}^{+} / \mathrm{APC}^{+}\right)$, respectively (Figure 4B). As a percentage of $\mathrm{BrdU}^{+}$cell density, lvMeteorin significantly increased the fraction of Olig2 ${ }^{+} / \mathrm{APC}^{-}$ oligodendrocyte progenitors $(8.1 \pm 0.8 \%)$ compared to lvGFP-treated animals ( $3.3 \pm 1 \%$; $p=0.04$, one-way ANOVA, Bonferroni post hoc), while there was no significant change between the lvGFP control group and the lvGDNF group $(5.1 \pm 2 \%)-$ Figure 4D. This equated to a significantly greater total number of $\mathrm{BrdU}^{+} / \mathrm{Olig}^{+} / \mathrm{APC}^{-}$oligodendrocyte progenitors in the anterior striatum of lvMeteorin treated animals $\left(8.3 \pm 0.8 \times 10^{3}\right.$ cells $)$, compared to the lvGFP $\left(3.1 \pm 0.9 \times 10^{3}\right.$ cells $)$ and lvGDNF groups $\left(6.2 \pm 2.2 \times 10^{3}\right.$ cells; lvGFP v lvMeteorin, $p=0.03$, one-way ANOVA, Bonferroni post hoc).

We then assessed whether the Meteorin mediated increase in newborn oligodendrocyte progenitors translated to a greater increase in differentiation toward post-mitotic $\left(\mathrm{APC}^{+}\right)$ oligodendrocytes. To do this, we assessed the fraction of $\mathrm{BrdU}^{+} / \mathrm{Olig}^{+}$cells that adopted an $\mathrm{APC}^{+}$phenotype. In the lvGFP group, an average of $13.4 \pm 4.3 \%$ of total $\mathrm{BrdU}^{+} / \mathrm{Olig}^{+}$ cells co-expressed APC. Comparison between treatment groups revealed that there was no significant difference between lvGFP animals and lvMeteorin $(8.0 \pm 1.5 \%)$ or lvGDNF animals (9.9 $\pm 3.1 \% ; p=0.71$, lvGFP v lvMeteorin; one-way ANOVA, Bonferroni post hoc) - Figure 4E. This result was consistent when comparing the average total $\mathrm{BrdU}^{+} / \mathrm{Olig}^{+} / \mathrm{APC}^{+}$cell number between groups (n.s., $p=0.55$, data not shown; lvGFP v lvMeteorin, one-way ANOVA, Bonferroni post hoc).

We assessed the effects of Meteorin treatment on microglia proliferation using the microglia marker Ibal in conjunction with BrdU (Figure 4F). In the lvGFP treatment group, $36.8 \pm 1.8 \%$ of $\mathrm{BrdU}^{+}$cells were $\mathrm{Ibal}^{+}$, indicating that this is the predominant phenotype of newborn cells postinjury (Figure 4G). To determine whether Meteorin or GDNF treatment influenced microglia proliferation after striatal injury, Iba1/BrdU quantification was conducted across these groups. Compared to the lvGFP control group, no significant difference was observed in the percentage of $\mathrm{Iba}^{+} / \mathrm{BrdU}^{+}$cells in the lvMeteorin groups $\left(49.3 \pm 8.2 \%\right.$ of $\mathrm{BrdU}^{+}$cells; $\left.p=0.27\right)$ and the lvGDNF group $\left(31.1 \pm 5 \%\right.$ of $\mathrm{BrdU}^{+}$cells; $p=0.72$; one-way ANOVA, Bonferroni post hoc) - Figure 4G indicating that these factors did not influence microglia proliferation post-striatal injury.

\section{DISCUSSION}

These results show that lentiviral delivery of Meteorin to the damaged striatum leads to robust over-expression of the diffusible protein resulting in increased gliogenesis but not 
A

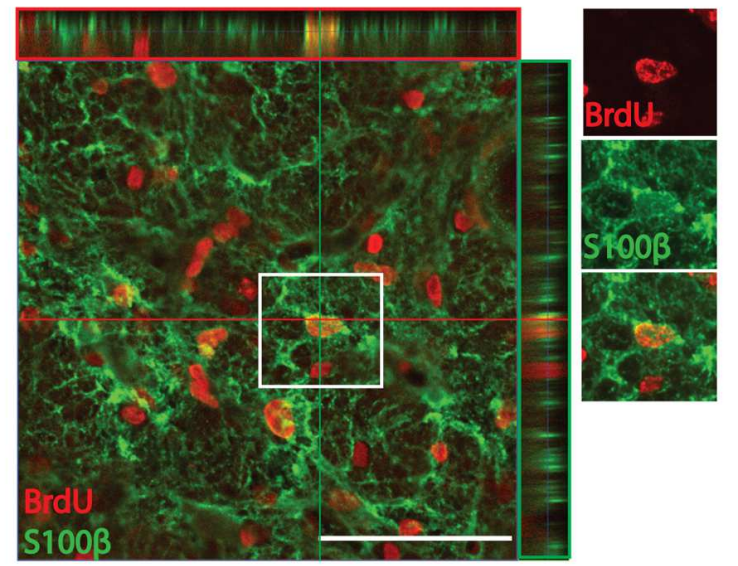

B

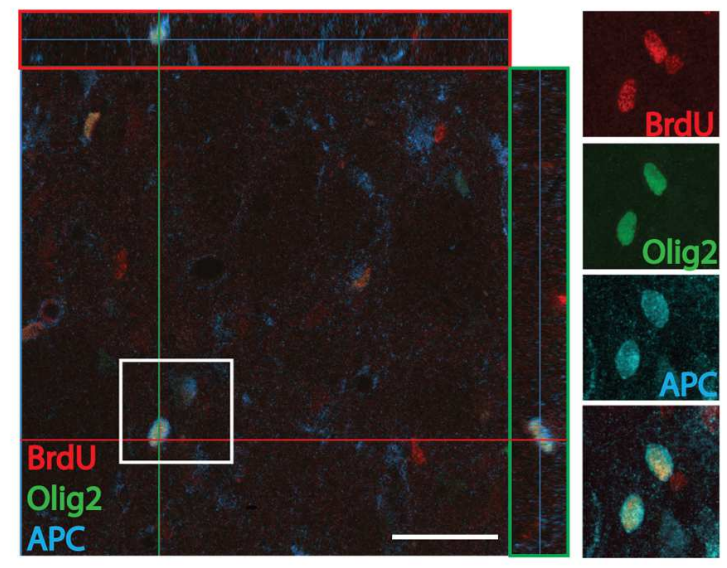

E
C

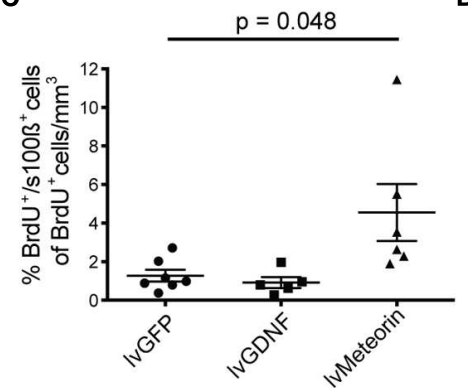

$\mathbf{F}$

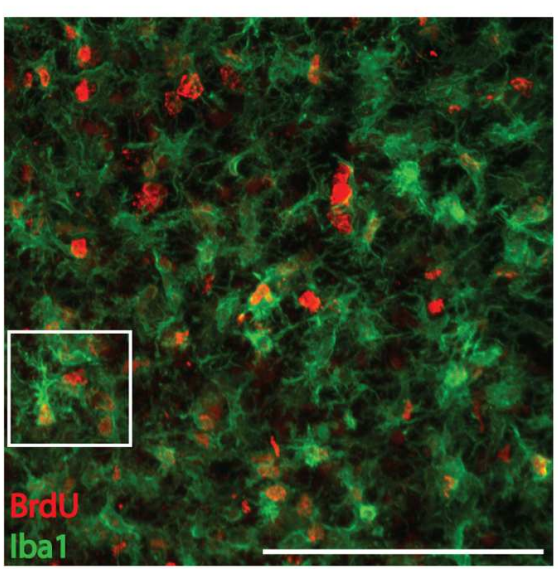

D

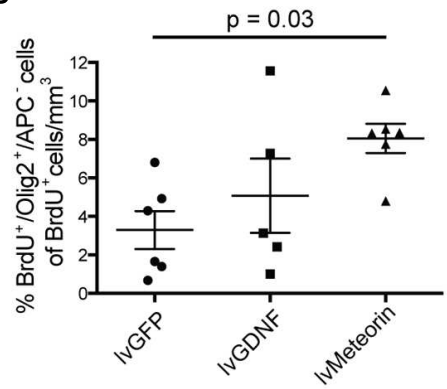

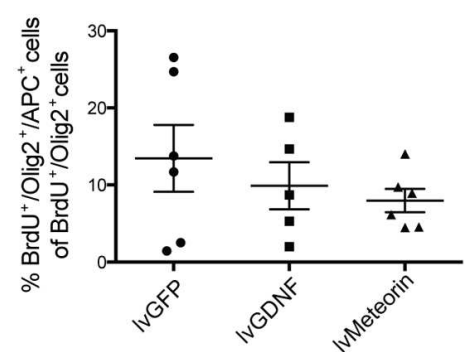

G
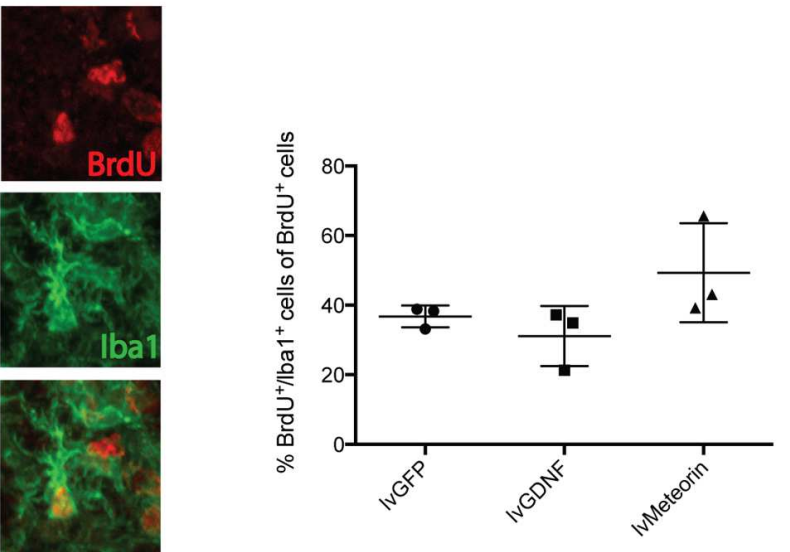

FIGURE 4 | Birth-dating of glial cells in the striatum after QA lesion and assessment of the effect of Meteorin on these populations. (A) Confocal orthogonal representation of cells in the striatum ipsilateral to lentiviral delivery and lesion confirm double labeling of $\mathrm{BrdU}^{+}$cells co-stained for either s100 $\beta$ to identify new born astrocytes or (B) Olig2 and APC to identify newborn OPCs (BrdU $+/ O$ lig2 $+/ \mathrm{APC}^{-}$cells) and mature oligodendrocytes $\left(\mathrm{BrdU}^{+} / \mathrm{Olig}^{+} / \mathrm{APC}^{+}\right.$cells; boxed insets detail double and triple labeling of the cells). Newborn glial cells were quantified and represented as a percentage of total BrdU ${ }^{+}$cell density. (C) Cell quantification of $\mathrm{BrdU}^{+} / \mathrm{s} 100 \beta^{+}$cells normalized to $\mathrm{BrdU}^{+}$cell density revealed IvMeteorin-treated animals had significantly more newborn s100 $\beta^{+}$astrocytes in the ipsilateral striatum compared to IVGFP controls. For Oligodendroglial lineages, (D) BrdU $+/ O l i g 2^{+} / \mathrm{APC}^{-}$(OPCs) revealed a significant increase in this population in IvMeteorin-treated animals compared to IvGFP-treated controls. (E) The percentage of BrdU+/Olig2 ${ }^{+}$cells that express APC (mature OLs) was assessed and was not significant between treatment groups. Oligodendrocyte lineage cell quantification was normalized to $\mathrm{BrdU}^{+}$cell density. (F) Representative BrdU/lba1 stain with example BrdU ${ }^{+} / \mathrm{lba} 1^{+}$cell (boxed) and (G) quantification of BrdU+/lba1+ cells as a percentage of total BrdU showed no significant difference between treatment groups. Data represents mean \pm SEM, IVGFP $n=7$; IVMeteorin $n=6$; IVGDNF $n=5$ for astrocyte and oligodendrocyte quantification and $n=3$ for microglia cell counts across all groups. Scale bar: $100 \mu \mathrm{m}$. OPC, Oligodendrocyte precursor cell; OL, Oligodendrocyte.

neurogenesis in response to striatal injury. Cell proliferation is a well-established response to acute brain injury (Cavanagh, 1970; Nait-Oumesmar et al., 1999) and it has been convincingly demonstrated that the majority of the newborn cells are local microglia (Marty et al., 1991; Amat et al., 1996) which we have shown accounts for $40 \%$ of newborn cells post-injury. 
Importantly, it has also been shown that, following damage to the striatum, a small proportion of the newborn cells at the site of injury are neurons that migrate from the adjacent SVZ (Arvidsson et al., 2001; Parent et al., 2002). This has led to the idea that augmentation of this neurogenic response might lead to regenerative therapies for brain repair.

A number of studies have significantly improved the level of injury-induced striatal neurogenesis through delivery of growth factors, either as protein infusions (Pencea et al., 2001; Kobayashi et al., 2006; Ninomiya et al., 2006) or using viral vectors (Chmielnicki et al., 2004; Benraiss et al., 2012; Yu et al., 2013). Here we chose to investigate Meteorin in this context based on previous work showing that it is up-regulated in the striatum following injury (Jorgensen et al., 2011; Wang et al., 2012; Lee et al., 2015), can protect striatal neurons from excitotoxic death (Jorgensen et al., 2011) and is a potent chemokinetic regulator of migratory doublecortin ${ }^{+}$neuroblasts in the striatum after middle cerebral artery occlusion (MCAO) (Wang et al., 2012). Although immunohistochemical analysis showed robust and diffuse over-expression of Meteorin throughout the lesioned striatum, there was no significant impact on the number of new striatal neurons generated in the first week after injury relative to the lvGFP control group. This is in contrast to a similar study recently reporting that Meteorin infused as a protein over 2 weeks following medial cerebral artery occlusion significantly increases the number of newborn neurons in the striatum by around $70 \%$ (Wang et al., 2012). The conflicting findings might be at least partly explained by experimental variables, including differences in the timing and dose of Meteorin delivery and also the severity of striatal damage. Notably, however, over-expression of GDNF using the same lentiviral construct delivered at the same time relative to injury resulted in a significant increase in the number of new neurons compared to the Meteorin and control groups.

We included a GDNF group in order to contextualize the impact of Meteorin. GDNF is well established as a neurotrophic factor in models of CNS injury and has previously been shown to increase the number of newborn cells that survive as mature neurons in the striatum after striatal injury (Kobayashi et al., 2006). We observed an 11-fold increase in the number of mature striatal neurons after striatal injury in GDNF treated animals, but no significant difference in Meteorin treated animals under the same experimental conditions. Collectively, the results suggest that the neurogenic impact of Meteorin following striatal damage is modest and may be relatively more sensitive to factors related to timing and dose as well as the nature of the injury. Understanding the efficacy of neurotrophic proteins in specific injury models is an important challenge for developing regenerative gene-based therapies for CNS injury.

Another potential role for neurotrophic factors in modulating the neurogenic response to injury is through impact on the differentiation of newborn cells toward therapeutically relevant cell types - for example striatal projection neurons that can replace those lost after damage. Based on immunohistochemistry for Darpp32 6 weeks after lesioning, no examples of new striatal projection neurons were found across any of the three treatment groups. However, a number of $\mathrm{BrdU}^{+} / \mathrm{NeuN}^{+}$cells were found to acquire a Calretinin ${ }^{+}$interneuron phenotype. These findings are consistent with a recent study showing that striatal injury can facilitate migration of nearby SVZ neuroblasts to the site of injury but does not influence their original, interneuron differentiation potential (De Marchis et al., 2007). Neither GDNF nor Meteorin influenced the fraction of $\mathrm{BrdU}^{+} / \mathrm{NeuN}^{+}$cells to adopt a Calretinin $^{+}$identity. Thus the mechanism underlying greater neuronal numbers in the GDNF group is likely to be related in increased survival rather than an influence on differentiation potential. We also observed many $\mathrm{BrdU}^{+} /$Calretinin ${ }^{+}$cells that did not express NeuN. This may represent a different Calretinin ${ }^{+}$ phenotype or reflect an immature status of these cells, as has been shown in studies on staging of protein expression in hippocampal neurogenesis (Brandt et al., 2003). It is important to note that the majority of $\mathrm{BrdU}^{+} / \mathrm{NeuN}^{+}$cells did not express Calretinin or Darpp32. This may reflect an immature phenotype within the newborn population of striatal neurons. However, previous studies have shown that insult-mediated neurogenesis in the striatum produces other interneuron phenotypes characterized by the markers parvalbumin and neuropeptide Y (Collin et al., 2005) which may also account for the remaining newborn neurons.

The most prominent impact of Meteorin over-expression on newborn cells generated after injury was a significant increase in the proportion of glial cell types, including $s 100 \beta^{+}$mature astrocytes. This is consistent with previous in vitro findings using primary cultures, where Meteorin has been shown to act through the Jak-STAT3 pathway to increase astrocyte differentiation (Nishino et al., 2004; Lee et al., 2010; Sun et al., 2014). We have previously reported that Meteorin is up-regulated in s $100 \beta$ cells at the site of injury after excitotoxic lesioning of the striatum. Taken together, these data suggest that Meteorin may act as a paracrine signaling molecule to drive gliosis via differentiation in response to brain damage as opposed to acting as a trophic factor for these cells. We also looked at microglial phenotype amongst newborn cells. Iba $1+$ microglia accounted for $\sim 40 \%$ of all newborn $\mathrm{BrdU}^{+}$ cells post injury. This is in line with previous literature which describes an early and rapid proliferative response of microglia after acute injury in the CNS (Morioka et al., 1993; Topper et al., 1993; Thored et al., 2009; Taylor and Sansing, 2013). The lack of impact on the proportion of $\mathrm{BrdU}^{+}$microglial cells suggests a specific function for Meteorin in astrocyte differentiation rather than a broad exacerbation of inflammatory response to injury.

Interestingly, Meteorin also increased the number of oligodendrocyte progenitor cells (OPCs). Here we report that Meteorin significantly increases the number of Olig2 ${ }^{+}$OPCs in the striatum following excitotoxic damage. This reveals a novel biological function for Meteorin in vivo and indicates therapeutic potential in pathological settings involving loss of oligodendrocytes-for example the demyelination of neurons resulting from loss of oligodendrocytes after ischemic stroke (Pantoni et al., 1996; Dewar et al., 2003). Notably, while the OPC pool increased in Meteorin treated animals, this did not translate to an increase in mature $\mathrm{APC}^{+}$oligodendrocytes. This may reflect that additional signaling components are required for maturation in the lesioned striatum and/or an unfavorable 
environment for differentiation. In future studies it will be valuable to assess and compare the oligogenic effects of Meteorin with other molecules reported to drive oligodendrogliogenesis after injury, such as Erythropoietin (Zhang et al., 2010) and Noggin (Irvin et al., 2008) as well as the mechanism driving this increase in OPCs to determine whether Meteorin influences the proliferation and differentiation of this population or offers trophic support to OPCs. Assessment of the timing and combinatorial delivery of these molecules may reveal an optimal approach for increasing both the number of oligodendrocytes and their capacity for myelination following de-myelinating injuries. Other valuable future studies to extend on the present findings would include lineage tracing work aimed at identifying the cells that respond to MTRN in order to drive gliogenesis. There is strong evidence to suggest that the neurogenic response in a variety of striatal injury models, including MCAO (Arvidsson et al., 2002), QA lesioning (Collin et al., 2005), and traumatic brain injury (Richardson et al., 2007) is largely provided by the nearby pool of neurogenic precursors in the SVZ. The origin of the gliogenic response is less well characterized but may include both an SVZ component - for example OPCs can be generated by SVZ precursors and migrate into the striatum following stroke (Li et al., 2010) - as well as the proliferation of local parenchymal progenitors or differentiated glia at the site of injury.

In summary, we demonstrated that Meteorin was able to increase astrogenesis in the striatum after acute injury characterized by widespread neuronal cell loss. We also revealed a novel function for Meteorin in significantly increasing the number of OPCs post-injury. Interestingly, Meteorin did not influence the maturation of these cells into mature

\section{REFERENCES}

Amat, J. A., Ishiguro, H., Nakamura, K., and Norton, W. T. (1996). Phenotypic diversity and kinetics of proliferating microglia and astrocytes following cortical stab wounds. Glia 16, 368-382. doi: 10.1002/(SICI)10981136(199604)16:4<368::AID-GLIA9>3.0.CO;2-W

Arvidsson, A., Collin, T., Kirik, D., Kokaia, Z., and Lindvall, O. (2002). Neuronal replacement from endogenous precursors in the adult brain after stroke. Nat. Med. 8, 963-970. doi: 10.1038/nm747

Arvidsson, A., Kokaia, Z., Airaksinen, M. S., Saarma, M., and Lindvall, O. (2001). Stroke induces widespread changes of gene expression for glial cell line-derived neurotrophic factor family receptors in the adult rat brain. Neuroscience 106, $27-41$.

Benraiss, A., Bruel-Jungerman, E., Lu, G., Economides, A. N., Davidson, B., and Goldman, S. A. (2012). Sustained induction of neuronal addition to the adult rat neostriatum by AAV4-delivered noggin and BDNF. Gene Ther. 19, 483-493. doi: 10.1038 /gt.2011.114

Brandt, M. D., Jessberger, S., Steiner, B., Kronenberg, G., Reuter, K., BickSander, A., et al. (2003). Transient calretinin expression defines early postmitotic step of neuronal differentiation in adult hippocampal neurogenesis of mice. Mol. Cell. Neurosci. 24, 603-613. doi: 10.1016/S1044-7431(03) 00207-0

Cavanagh, J. B. (1970). The proliferation of astrocytes around a needle wound in the rat brain. J. Anat. 106, 471-487.

Chmielnicki, E., Benraiss, A., Economides, A. N., and Goldman, S. A. (2004). Adenovirally expressed noggin and brain-derived neurotrophic factor cooperate to induce new medium spiny neurons from resident progenitor cells in the adult striatal ventricular zone. J. Neurosci. 24, 2133-2142. doi: 10.1523/JNEUROSCI.1554-03.2004 oligodendrocytes, suggesting a discrete function on the OPC population. Meteorin was not effective for augmentation of the neurogenic response to injury. These findings highlight that development of strategies for brain-repair based on trophicfactor delivery will likely require combinatorial approaches, where specific proteins are targeted to affect the survival and differentiation of specific cell types.

\section{AUTHOR CONTRIBUTIONS}

JW, LT, and CP performed surgical procedures. JW processed, imaged, and quantified the tissue obtained. CE acquired representative images and JJ constructed the lentiviral vectors for gene delivery. LT funded and contributed to the design and analysis of the study.

\section{FUNDING}

CP is a Viertel Senior Research Fellow. This work was supported NHMRC project grant \#1042584. The Florey Institute of Neuroscience and Mental Health acknowledges the strong support from the Victorian Government and in particular the funding from the Operational Infrastructure Support Grant.

\section{ACKNOWLEDGMENT}

The authors thank Mong Tien for expert technical assistance in the tissue preparation and immunohistochemical procedures.

Collin, T., Arvidsson, A., Kokaia, Z., and Lindvall, O. (2005). Quantitative analysis of the generation of different striatal neuronal subtypes in the adult brain following excitotoxic injury. Exp. Neurol. 195, 71-80. doi: 10.1016/j.expneurol.2005.03.017

De Marchis, S., Bovetti, S., Carletti, B., Hsieh, Y. C., Garzotto, D., Peretto, P., et al. (2007). Generation of distinct types of periglomerular olfactory bulb interneurons during development and in adult mice: implication for intrinsic properties of the subventricular zone progenitor population. J. Neurosci. 27, 657-664. doi: 10.1523/JNEUROSCI.2870-06.2007

Dewar, D., Underhill, S. M., and Goldberg, M. P. (2003). Oligodendrocytes and ischemic brain injury. J. Cereb. Blood Flow Metab. 23, 263-274.

Gundersen, H. J., Bagger, P., Bendtsen, T. F., Evans, S. M., Korbo, L., Marcussen, N., et al. (1988). The new stereological tools: disector, fractionator, nucleator and point sampled intercepts and their use in pathological research and diagnosis. APMIS 96, 857-881. doi: 10.1111/j.1699-0463.1988.tb00954.x

Irvin, D. K., Kirik, D., Bjorklund, A., and Thompson, L. H. (2008). In vivo gene delivery to proliferating cells in the striatum generated in response to a 6hydroxydopamine lesion of the nigro-striatal dopamine pathway. Neurobiol. Dis. 30, 343-352. doi: 10.1016/j.nbd.2008.02.006

Jin, K., Minami, M., Lan, J. Q., Mao, X. O., Batteur, S., Simon, R. P., et al. (2001). Neurogenesis in dentate subgranular zone and rostral subventricular zone after focal cerebral ischemia in the rat. Proc. Natl. Acad. Sci. U.S.A. 98, 4710-4715. doi: 10.1073/pnas.081011098

Jin, K., Sun, Y., Xie, L., Peel, A., Mao, X. O., Batteur, S., et al. (2003). Directed migration of neuronal precursors into the ischemic cerebral cortex and striatum. Mol. Cell. Neurosci. 24, 171-189.

Jin, K., Wang, X., Xie, L., Mao, X. O., Zhu, W., Wang, Y., et al. (2006). Evidence for stroke-induced neurogenesis in the human brain. Proc. Natl. Acad. Sci. U.S.A. 103, 13198-13202. doi: 10.1073/pnas.0603512103 
Jorgensen, J. R., Emerich, D. F., Thanos, C., Thompson, L. H., Torp, M., Bintz, B., et al. (2011). Lentiviral delivery of meteorin protects striatal neurons against excitotoxicity and reverses motor deficits in the quinolinic acid rat model. Neurobiol. Dis. 41, 160-168. doi: 10.1016/j.nbd.2010.09.003

Jorgensen, J. R., Thompson, L., Fjord-Larsen, L., Krabbe, C., Torp, M., Kalkkinen, N., et al. (2009). Characterization of Meteorin-an evolutionary conserved neurotrophic factor. J. Mol. Neurosci. 39, 104-116. doi: 10.1007/s12031-009-9189-4

Kobayashi, T., Ahlenius, H., Thored, P., Kobayashi, R., Kokaia, Z., and Lindvall, O. (2006). Intracerebral infusion of glial cell line-derived neurotrophic factor promotes striatal neurogenesis after stroke in adult rats. Stroke 37, 2361-2367. doi: 10.1161/01.STR.0000236025.44089.e1

Lee, H. S., Han, J., Lee, S. H., Park, J. A., and Kim, K. W. (2010). Meteorin promotes the formation of GFAP-positive glia via activation of the Jak-STAT3 pathway. J. Cell Sci. 123, 1959-1968. doi: 10.1242/jcs.063784

Lee, H. S., Lee, S. H., Cha, J. H., Seo, J. H., Ahn, B. J., and Kim, K. W. (2015). Meteorin is upregulated in reactive astrocytes and functions as a negative feedback effector in reactive gliosis. Mol. Med. Rep. 12, 1817-1823. doi: 10.3892/mmr.2015.3610

Li, L., Harms, K. M., Ventura, P. B., Lagace, D. C., Eisch, A. J., and Cunningham, L. A. (2010). Focal cerebral ischemia induces a multilineage cytogenic response from adult subventricular zone that is predominantly gliogenic. Glia 58, 16101619. doi: $10.1002 /$ glia.21033

Marty, S., Dusart, I., and Peschanski, M. (1991). Glial changes following an excitotoxic lesion in the CNS-I. Microglia/macrophages. Neuroscience 45, 529539. doi: 10.1016/0306-4522(91)90268-S

Mayhew, T. M. (1991). The new stereological methods for interpreting functional morphology from slices of cells and organs. Exp. Physiol. 76, 639-665. doi: 10.1113/expphysiol.1991.sp003533

Morioka, T., Kalehua, A. N., and Streit, W. J. (1993). Characterization of microglial reaction after middle cerebral artery occlusion in rat brain. J. Comp. Neurol. 327, 123-132. doi: 10.1002/cne.903270110

Nait-Oumesmar, B., Decker, L., Lachapelle, F., Avellana-Adalid, V., Bachelin, C., and Baron-Van Evercooren, A. (1999). Progenitor cells of the adult mouse subventricular zone proliferate, migrate and differentiate into oligodendrocytes after demyelination. Eur. J. Neurosci. 11, 4357-4366.

Ninomiya, M., Yamashita, T., Araki, N., Okano, H., and Sawamoto, K. (2006). Enhanced neurogenesis in the ischemic striatum following EGF-induced expansion of transit-amplifying cells in the subventricular zone. Neurosci. Lett. 403, 63-67. doi: 10.1016/j.neulet.2006.04.039

Nishino, J., Yamashita, K., Hashiguchi, H., Fujii, H., Shimazaki, T., and Hamada, H. (2004). Meteorin: a secreted protein that regulates glial cell differentiation and promotes axonal extension. EMBO J. 23, 1998-2008. doi: 10.1038/sj.emboj.7600202

Pantoni, L., Garcia, J. H., and Gutierrez, J. A. (1996). Cerebral white matter is highly vulnerable to ischemia. Stroke 27, 1641-1646; discussion 1647.

Parent, J. M., Vexler, Z. S., Gong, C., Derugin, N., and Ferriero, D. M. (2002). Rat forebrain neurogenesis and striatal neuron replacement after focal stroke. Ann. Neurol. 52, 802-813. doi: 10.1002/ana.10393

Paxinos, G., and Watson, C. (1998). The Rat Brain in Stereotaxic Coordinates, 4th Edn. Cambridge, MA: Academic Press.

Pencea, V., Bingaman, K. D., Wiegand, S. J., and Luskin, M. B. (2001). Infusion of brain-derived neurotrophic factor into the lateral ventricle of the adult rat leads to new neurons in the parenchyma of the striatum, septum, thalamus, and hypothalamus. J. Neurosci. 21, 6706-6717.

Richardson, R. M., Sun, D., and Bullock, M. R. (2007). Neurogenesis after traumatic brain injury. Neurosurg. Clin. N. Am. 18, 169-181. doi: 10.1016/j.nec.2006.10.007

Sun, Y., Jin, K., Xie, L., Childs, J., Mao, X. O., Logvinova, A., et al. (2003). VEGFinduced neuroprotection, neurogenesis, and angiogenesis after focal cerebral ischemia. J. Clin. Invest. 111, 1843-1851. doi: 10.1172/JCI17977

Sun, Y., Lehmbecker, A., Kalkuhl, A., Deschl, U., Sun, W., Rohn, K., et al. (2014). STAT3 represents a molecular switch possibly inducing astroglial instead of oligodendroglial differentiation of oligodendroglial progenitor cells in Theiler's murine encephalomyelitis. Neuropathol. Appl. Neurobiol. 41, 347-370. doi: $10.1111 /$ nan. 12133

Taylor, R. A., and Sansing, L. H. (2013). Microglial responses after ischemic stroke and intracerebral hemorrhage. Clin. Dev. Immun. 2013:746068. doi: $10.1155 / 2013 / 746068$

Thored, P., Heldmann, U., Gomes-Leal, W., Gisler, R., Darsalia, V., Taneera, J., et al. (2009). Long-term accumulation of microglia with proneurogenic phenotype concomitant with persistent neurogenesis in adult subventricular zone after stroke. Glia 57, 835-849. doi: 10.1002/glia.20810

Topper, R., Gehrmann, J., Schwarz, M., Block, F., Noth, J., and Kreutzberg, G. W. (1993). Remote microglial activation in the quinolinic acid model of Huntington's disease. Exp. Neurol. 123, 271-283. doi: 10.1006/exnr.1993.1159

Tornoe, J., Torp, M., Jorgensen, J. R., Emerich, D. F., Thanos, C., Bintz, B., et al. (2012). Encapsulated cell-based biodelivery of meteorin is neuroprotective in the quinolinic acid rat model of neurodegenerative disease. Restor. Neurol. Neurosci. 30, 225-236. doi: 10.3233/RNN-2012-110199

Wang, Z., Andrade, N., Torp, M., Wattananit, S., Arvidsson, A., Kokaia, Z., et al. (2012). Meteorin is a chemokinetic factor in neuroblast migration and promotes stroke-induced striatal neurogenesis. J. Cereb. Blood Flow Metab. 32, 387-398. doi: $10.1038 /$ jcbfm.2011.156

West, M. J., Slomianka, L., and Gundersen, H. J. (1991). Unbiased stereological estimation of the total number of neurons in thesubdivisions of the rat hippocampus using the optical fractionator. Anat. Rec. 231, 482-497. doi: 10.1002/ar.1092310411

Yu, S. J., Tseng, K. Y., Shen, H., Harvey, B. K., Airavaara, M., and Wang, Y. (2013). Local administration of AAV-BDNF to subventricular zone induces functional recovery in stroke rats. PLoS ONE 8:e81750. doi: 10.1371/journal.pone.0081750

Zhang, L., Chopp, M., Zhang, R. L., Wang, L., Zhang, J., Wang, Y., et al. (2010). Erythropoietin amplifies stroke-induced oligodendrogenesis in the rat. PLoS ONE 5:e11016. doi: 10.1371/journal.pone.0011016

Conflict of Interest Statement: The authors declare that the research was conducted in the absence of any commercial or financial relationships that could be construed as a potential conflict of interest.

Copyright (c) 2016 Wright, Ermine, Jørgensen, Parish and Thompson. This is an open-access article distributed under the terms of the Creative Commons Attribution License (CC BY). The use, distribution or reproduction in other forums is permitted, provided the original author(s) or licensor are credited and that the original publication in this journal is cited, in accordance with accepted academic practice. No use, distribution or reproduction is permitted which does not comply with these terms. 